\title{
The Biological Basis of Social Cognition During Development
}

Over the past two decades, there has been a major growth in publications related to the development and biological basis of social cognitive processes. An increasingly diverse range of cognitive neuroscience techniques, including EEG and fNIRS, have been deployed in order to understand aspects of social functioning during infancy and childhood. There are also an increasing number of researchers who are assuming a lifespan perspective. The aim of this special issue is to showcase cutting edge research in this field across an array of areas with a diversity of neuroscience methods.

The first section is concerned with a fundamental aspect of social perception, namely the neural processing of faces in the developing brain. Given the long-standing debate on whether specialized face processing in the adult human brain is mainly due to domain-specific mechanisms (McKone et al., 2007) or quantitative differences in expertise acquired for faces compared to other visual stimuli across development (Gauthier and Bukach, 2007), a look into the neural mechanisms of face perception in early childhood yields important new insights. Timeo et al. (2017) applied fNIRS to study the perception of Caucasian and African faces in 5- to 9-month-old Caucasian infants. They report progressive specialization across the two age groups. Further probing hemispheric specialization, Lochy et al. (2017) use fast periodic visual stimulation (FPVS) to show that preschoolers, unlike infants, do not show a clear right hemispheric lateralization of neural responses to human face stimuli. These results speak to non-linear development of right hemisphere specialization across childhood. Munsters et al. (2017) present evidence of adequate test-retest reliability of face-sensitive event-related potential (ERP) components derived from the EEG signal in 9- to 10-month-old infants across two test sessions. However, the effects of certain stimulus manipulations (emotions and spatial frequency) were not stable across measurement time points, calling into question whether differential responses can reliably be seen as indicative of individual developmental trajectories. Dixon et al. (2017) focus on the effects of exposure to other-species faces on categorization and individuation in 9-month-olds' ERP responses. Whereas exposure to multiple exemplars facilitated infant categorization of monkey faces on the subordinate level, familiarization to an individual monkey face did not affect infant categorization or individuation of monkey faces, speaking to the specific effects of exposure to multiple exemplars of an unfamiliar face category on infant face perception. Finally, Proverbio and De Gabriele (2017) show that the well-known other-race effect does not apply to infant faces in adult participants.

Important social information can be derived from faces. Michel and colleagues focused on infants' direction of attention through social cues and show that eye-like stimuli are necessary for an unfamiliar object to effectively direct 4-month-olds' attention toward cued peripheral stimuli. Jessen and Grossmann report evidence for the subliminal processing of facial trustworthiness already in 7-month-old infants. Regarding emotional facial expressions, van den Boomen et al. (2017) tested 9- to 10-month-olds ERP responses to happy, fearful, and neutral faces containing either high or low spatial frequency information. Whereas face-specific posterior components only discriminated between emotions when high spatial frequency information was given, the attention-related Nc showed differential responses to emotions regardless of spatial frequency.

Above and beyond early face perception, infants' processing of and understanding of other people's actions is a vital field of research in the domain of social cognition in development. Linnert, Tóth, Nagy, Parise, and Király (2017) found larger amplitude of the Nc to objects that were previously presented by a hand (familiarized stimuli) compared to the novel ones in 10- to 12-month-old infants, suggesting that the $\mathrm{Nc}$ is an ERP signature of recognition memory, a crucial component of social and cognitive development. Reid et al. (2017) provide ERP evidence for a link between action production and action perception already in 10week-old infants, speaking for effective motor resonance in early infancy. This finding is corroborated by results of Antognini and Daum (2017) who demonstrate sensorimotor oscillatory activity during auditory verb processing in toddlers. Monroy et al. (2017) use ERPs to show that action-effect associations help 8- to 11-month-old infants to learn regularities in action sequences. Schönebeck and Elsner (2017) showed that both earlier and later ERP components in 14-month-old infants are sensitive to incomplete end-states of goal-directed actions. This speaks for the use of bottom-up perceptual as well as top-down conceptual processing in action perception in the second year.

In addition to face perception and action understanding, contributions of this special issue are devoted to social behavior. Hepach et al. (2017) show that children's physiological arousal, assessed through pupil dilation measures, predicts their helping behavior, thus, shedding light on the biological mechanisms underlying young children's prosocial motivations. van Wijk et al. (2017) report a substantial relation between negative (vs. positive) social judgments and aggressive behavior. They did not find evidence for a mediating effect of frontal EEG asymmetry, highlighting that further research is necessary to pinpoint the neural correlates of this effect.

The final section of this special issue is dedicated to atypical development and impairments in social cognition. Luyster et al. (2017) report differences in the latencies of visual ERP components to emotional faces in children and young adults with autism spectrum disorder and a typically developing control group speaking to differential maturation of cortical networks underlying emotional face processing in the two groups. Using both face and body expressions of different emotions, Malaia et al. (2017) also report differences in emotional processing between children with autism and their typically developing peers. Regarding atypical language development, Schaadt et al. (2018) 
find that children with developmental dyslexia show reduced sensitivity for visual-auditory speech perception, suggesting that they might benefit from early bimodal speech training. Finally, Baez et al. (2017) report grey matter atrophy associated with executive function and social cognition deficits in behavioral variant frontotemporal dementia.

Taken together, the original work presented in this special issue showcases the richness of research questions and the diversity of methods used in current research on social cognitive development in healthy as well as in impaired populations across the lifespan. A common denominator of the presented research is that neuroscience methods can yield invaluable new insights into social cognitive development. As this special issue shows, this is clearly a field that is rapidly growing and benefits from a plurality of methods and perspectives.

\section{References}

Antognini, K., Daum, M.M., 2017. Toddlers show sensorimotor activity during auditory verb processing. Neuropsychologia. https://doi.org/10.1016/j.neuropsychologia. 2017.07.022.

Baez, S., Pinasco, C., Roca, M., Ferrari, J., Couto, B., Garcia-Cordero, I., Torralva, T., et al., 2017. Brain structural correlates of executive and social cognition profiles in behavioral variant frontotemporal dementia and elderly bipolar disorder. Neuropsychologia. https://doi.org/10.1016/j.neuropsychologia.2017.02.012.

Dixon, K.C., Reynolds, G.D., Romano, A.C., Roth, K.C., Stumpe, A.L., Guy, M.W., Mosteller, S.M., 2017. Neural correlates of individuation and categorization of otherspecies faces in infancy. Neuropsychologia. https://doi.org/10.1016/j neuropsychologia.2017.09.037.

Gauthier, I., Bukach, C., 2007. Should we reject the expertise hypothesis? Cognition 103 (2), 322-330. https://doi.org/10.1016/j.cognition.2006.05.003. pii:S0010-0277(06) 00083-7.

Hepach, R., Vaish, A., Muller, K., Tomasello, M., 2017. The relation between young children's physiological arousal and their motivation to help others. Neuropsychologia. https://doi.org/10.1016/j.neuropsychologia.2017.10.010.

Linnert, S., Tóth, B., Nagy, M., Parise, E., Király, I., 2017. Neural signatures of recognition memory in 10- to 12-month-old infants. Neuropsychologia. https://doi.org/10.1016/ j.neuropsychologia.2017.08.023.

Lochy, A., de Heering, A., Rossion, B., 2017. The non-linear development of the right hemispheric specialization for human face perception. Neuropsychologia. https:// doi.org /10.1016/j.neuropsychologia.2017.06.029.

Luyster, R.J., Bick, J., Westerlund, A., Nelson 3rd, C.A., 2017. Testing the effects of expression, intensity and age on emotional face processing in ASD Neuropsychologia. https://doi.org/10.1016/j.neuropsychologia.2017.06.023.

Malaia, E., Cockerham, D., Rublein, K., 2017. Visual integration of fear and anger emotional cues by children on the autism spectrum and neurotypical peers: An EEG study. Neuropsychologia. https://doi.org/10.1016/j.neuropsychologia.2017.06.014.

McKone, E., Kanwisher, N., Duchaine, B.C., 2007. Can generic expertise explain special processing for faces? Trends in Cognitive Sciences 11 (1), 8-15. https://doi.org/10. 1016/j.tics.2006.11.002. pii:S1364-6613(06)00300-7.

Monroy, C.D., Gerson, S.A., Dominguez-Martinez, E., Kaduk, K., Hunnius, S., Reid, V. 2017. Sensitivity to structure in action sequences: An infant event-related potential study. Neuropsychologia. https://doi.org/10.1016/j.neuropsychologia.2017.05.007.

Munsters, N.M., van Ravenswaaij, H., van den Boomen, C., Kemner, C., 2017. Test-retest reliability of infant event related potentials evoked by faces. Neuropsychologia. https://doi.org/10.1016/j.neuropsychologia.2017.03.030.

Proverbio, A.M., De Gabriele, V., 2017. The other-race effect does not apply to infant faces: An ERP attentional study. Neuropsychologia. https://doi.org/10.1016/j. neuropsychologia.2017.03.028.

Reid, V.M., Kaduk, K., Lunn, J., 2017. Links between action perception and action production in 10-week-old infants. Neuropsychologia. https://doi.org/10.1016/j. neuropsychologia. 2017.11.005.

Schaadt, G., van der Meer, E., Pannekamp, A., Oberecker, R., Männel, C., 2018. Children with dyslexia show a reduced processing benefit from bimodal speech information compared to their typically developing peers. Neuropsychologia. https://doi.org/10 1016/j.neuropsychologia.2018.01.013.

Schönebeck, M., Elsner, B., 2017. ERPs reveal perceptual and conceptual processing in 14-month-olds' observation of complete and incomplete action end-states. Neuropsychologia. https://doi.org/10.1016/j.neuropsychologia.2017.10.026.

Timeo, S., Brigadoi, S., Farroni, T., 2017. Perception of Caucasian and African faces in 5to 9-month-old Caucasian infants: A functional near-infrared spectroscopy study. Neuropsychologia. https://doi.org/10.1016/j.neuropsychologia.2017.09.011.

van den Boomen, C., Munsters, N.M., Kemner, C., 2017. Emotion processing in the infant brain: The importance of local information. Neuropsychologia. https://doi.org/10. 1016/j.neuropsychologia.2017.09.006.

van Wijk, I.C., van den Bulk, B.G., Euser, S., Bakermans-Kranenburg, M.J., van, I.M.H., Huffmeijer, R., 2017. Social judgments, frontal asymmetry, and aggressive behavior in young children: A replication study using EEG. Neuropsychologia. https://doi.org/ 10.1016/j.neuropsychologia.2017.06.022.

Stefanie Hoehl

University of Vienna, Faculty of Psychology

Vincent M. Reid, Eugenio Parise Lancaster University, Department of Psychology 